\title{
Exhaled volatile organic compounds in adult asthma: a systematic review
}

\author{
Adnan Azim (1) ${ }^{1,2}$, Clair Barber $\mathbb{1}^{1,2}$, Paddy Dennison ${ }^{2}$, John Riley ${ }^{3}$ and \\ Peter Howarth ${ }^{1,2}$
}

Affiliations: ${ }^{1}$ Clinical and Experimental Sciences, Faculty of Medicine, University of Southampton, Southampton, UK. ${ }^{2}$ National Institute for Health Research (NIHR) Southampton Biomedical Research Centre, University Hospital Southampton NHS Foundation Trust, Southampton, UK. ${ }^{3}$ Galaxy Asthma, GSK, Medicines Research Centre, Stevenage, UK.

Correspondence: Adnan Azim, Clinical Research Fellow, Asthma, Allergy and Clinical Immunology, Mailpoint 52 , Floor 2 Minerva House, Southampton General Hospital, Tremona Road, Southampton, Hampshire, S016 6YD, UK. E-mail: a.azimdasoton.ac.uk

@ERSpublications

The clinical utility of exhaled breath sampling contrasts the complexity required for VOC analysis. Modern studies are now starting to apply robust methodological standards demonstrating breathomics to be sensitive to underlying inflammation. http://bit.ly/2WS1Sfx

Cite this article as: Azim A, Barber C, Dennison P, et al. Exhaled volatile organic compounds in adult asthma: a systematic review. Eur Respir J 2019; 54: 1900056 [https://doi.org/10.1183/13993003.00056-2019].

ABSTRACT The search for biomarkers that can guide precision medicine in asthma, particularly those that can be translated to the clinic, has seen recent interest in exhaled volatile organic compounds (VOCs). Given the number of studies reporting "breathomics" findings and its growing integration in clinical trials, we performed a systematic review of the literature to summarise current evidence and understanding of breathomics technology in asthma.

A PRISMA (Preferred Reporting Items for Systematic Reviews and Meta-Analyses)-oriented systematic search was performed (CRD42017084145) of MEDLINE, Embase and the Cochrane databases to search for any reports that assessed exhaled VOCs in adult asthma patients, using the following terms (asthma AND (volatile organic compounds AND exhaled) OR breathomics).

Two authors independently determined the eligibility of 2957 unique records, of which 66 underwent full-text review. Data extraction and risk of bias assessment was performed on the 22 studies deemed to fulfil the search criteria. The studies are described in terms of methodology and the evidence narratively summarised under the following clinical headings: diagnostics, phenotyping, treatment stratification, treatment monitoring and exacerbation prediction/assessment.

Our review found that most studies were designed to assess diagnostic potential rather than focus on underlying biology or treatable traits. Results are generally limited by a lack of methodological standardisation and external validation and by insufficiently powered studies, but there is consistency across the literature that exhaled VOCs are sensitive to underlying inflammation. Modern studies are applying robust breath analysis workflows to large multi-centre study designs, which should unlock the full potential of measurement of exhaled volatile organic compounds in airways diseases such as asthma.

This article has supplementary material available from erj.ersjournals.com

Received: 08 Jan 2019 | Accepted after revision: 13 June 2019

Copyright $\odot$ ERS 2019 


\section{Introduction}

In observing the phenotypic heterogeneity of the asthma population $[1,2]$ and its varied response to currently available treatments [3-5], it is clear that asthma describes a spectrum of biological complexity. Rather than diagnostics, the challenge for clinicians and researchers is the accurate stratification of patients based upon their underlying physiology/pathophysiology and the identification of treatable traits $[6,7]$. Critical to this ambition of precision medicine is the identification of biomarkers. Those biomarkers that can be easily sampled at the point of care have the highest translational potential [8]. In this regard, the clinical adoption of carbon-13/14 urea for Helicobacter pylori [9] and exhaled nitric oxide fraction (FeNO) for airway inflammation [10] demonstrates the utility of exhaled breath: this medium can be sampled safely, non-invasively and repeatedly, almost without exhaustion (e.g. capnography [11]). Moreover, through contact with the airways, exhaled breath measurements can potentially offer a direct insight into the organ of interest [12].

Although primarily composed of water vapour and inert gases, exhaled breath also contains thousands of volatile organic compounds (VOCs) [13]. VOCs are the main molecular substrate triggering our sense of smell, and characteristic breath odours have been used to identify illnesses since Hippocrates [14]. The medical applications of this strategy were transformed in 1971 by the demonstration that exhaled breath contained $>250$ VOCs [15]. We now recognise that changes in exhaled VOC concentrations can reflect different disease states [16, 17], suggesting a role for exhaled VOC analysis in non-invasive and early diagnostics [14].

Modern VOC analysis can be considered under one of two broad methodological headings: pattern recognition based sensors or chemical analytical techniques [18]. Pattern recognition based sensors, synonymous with electronic noses, are modelled on the mammalian nose. These e-noses contain an array of cross-reactive sensors, which react promiscuously and non-selectively to VOCs. The final "breathprint" reflects the differential signalling of multiple sensors to partially overlapping VOCs [19, 20]. These breathprints then require analysis by pattern recognition algorithms [21, 22], similar to how our brain would interpret smells. Chemical analytical techniques typically refer to mass spectrometry (MS) [23] or MS hybrid techniques [24-26], in which ions created by VOCs can be measured based upon their mass/charge $(\mathrm{m} / \mathrm{z})$ ratio.

In asthma, exhaled VOC analysis has demonstrated excellent accuracy for discriminating patients from healthy controls and other respiratory conditions [27]. The purpose of this systematic review is to describe the evidence for exhaled VOCs as a biomarker not only for diagnostics, but also other major clinical dilemmas faced by adult asthma clinicians: phenotyping, treatment stratification, treatment monitoring and exacerbation prediction/assessment [28]. We discuss these findings as well as methodologies surrounding breath sampling and VOC analysis; the challenges faced by this technology and future directions for research.

\section{Methods}

Search strategy and information sources

A PRISMA (Preferred Reporting Items for Systematic Reviews and Meta-Analyses)-oriented systematic search (CRD42017084145) (the full protocol can be accessed at www.crd.york.ac.uk/PROSPERO/) was performed until December 5, 2018 in Embase, MEDLINE and Cochrane Libraries. The search was conducted using keywords for "asthma", "volatile organic compounds", "exhaled" and "breathomics" and full Boolean operator terms (described in detail in the supplementary material). English-language peer-reviewed full-text articles were assessed for eligibility.

\section{Eligibility}

The ideal study for our review would prospectively recruit patients with asthma, diagnosed according to internationally recognised guidelines [29] and measure exhaled VOCs consistent with recently defined recommendations [30] including well-defined cut-off values against adequate reference standards. However, this would be difficult to define due to clinical and technological evolution over the timespan of the review. Similarly, the ideal study design for the detection and validation of exhaled VOC markers would be different for each of the clinical dilemmas that we wished to explore (diagnostics, phenotyping, treatment stratification, treatment monitoring and exacerbation prediction/assessment); thus, we used liberal inclusion criteria.

Studies were therefore included if 1) they measured exhaled breath $\operatorname{VOC}(\mathrm{s})$ in 2) a distinct group or patients with asthma defined by a trained physician or according to Global Initiative for Asthma or the American Thoracic Society/European Respiratory Society guidelines. Studies were excluded if they looked at non-adult (i.e. age $<18$ years) asthma patients; due to differences in diagnostic criteria between children and adults, as well as an attempt not to overlap with a recent review in paediatric populations [31]. 


\section{Study selection}

The titles and abstracts of all the records returned by the literature search were independently reviewed by two reviewers, to identify potentially relevant studies. The reviewers used the web-based software platform Rayyan (https://rayyan.qcri.org/) for screening and reviewing. Searches of study bibliographies were conducted to identify additional studies. Using the prespecified inclusion/exclusion criteria, these two reviewers then independently reviewed the full texts of potentially relevant studies to select trials for inclusion in the review. Disagreement was resolved by discussion, but in the event of non-consensus, it was planned that a third reviewer would be involved.

\section{Data extraction}

We extracted information from each study regarding 1) study design, 2) population, 3) breath collection methodology, 4) statistical analysis and 5) results.

\section{Risk of bias}

Risk of bias for each included study was assessed using the quality assessment of diagnostic accuracy studies (QUADAS)-2 tool [32]. The tool assesses the risk of bias across four domains: 1) patient selection, 2) index test, 3) reference standard and 4) flow/timing. Applicability is assessed across the first three domains.

\section{Method of analysis}

Studies were qualitatively assessed according to their methodology and results.

\section{Results}

\section{Search results}

Our search strategy yielded 2955 unique titles from Embase, MEDLINE and the Cochrane Library and two further titles through correspondence with authors in the field. Of these, 2891 were excluded after title and abstract review, leaving 66 full-text articles for screening (full-text exclusions are listed in the supplementary material). Reference list searching yielded no new studies. 22 articles were deemed to fulfil the inclusion/exclusion criteria (figure 1). All articles were assessed for risk of bias using the QUADAS-2 scoring system.

\section{Study characteristics}

Across these 22 studies, 1409 patients with asthma were investigated. The majority were recruited from European centres with just four studies recruiting from the USA [33], Australia [34, 35] and New Zealand [36].

Almost two-thirds $(61.0 \%)$ came from three studies [21, 37, 38]: the median sample size of studies was 25. As per the inclusion criteria, five studies only required a physician diagnosis of asthma while the other 18 specifically met recognised international diagnostic guidelines. Six studies used oral corticosteroid use as an exclusion criterion and 10 used smoking history.

The broad inclusion criteria of our search meant that several study designs were observed. Most studies employed a cross-sectional design with six including a longitudinal component. One study was purely correlational [39], but the remaining offered comparisons between groups (table 1). Many of these studies address more than one of the aforementioned clinical dilemmas (e.g. diagnosis and phenotyping) (table 1).

\section{Diagnosis}

In this review, we identified 14 studies which used VOC signals to discriminate asthma from other disease states; most often healthy controls, although four studies compared to a chronic obstructive pulmonary disease (COPD) group and one to lung cancer. One further study specifically compared asthma with allergic rhinitis to allergic rhinitis alone [44]. Notably, a study of 27 mild asthma patients found e-nose to outperform FeNO and simple spirometry in distinguishing asthma from healthy controls [42].

\section{Phenotype}

Six studies investigated the relationship between exhaled VOCs and airway inflammation in patients with asthma. Exhaled VOCs, identified using gas chromatography-mass spectrometry (GCMS), that were discriminatory between baseline and loss of control (following steroid withdrawal) [50] and which contributed to classification (of sputum cellular phenotype) models were found to correlate with sputum eosinophils [45]. Two studies used e-nose VOC profiles to correlate to bronchoalveolar lavage (BAL) and sputum eosinophil percentages finding an $\mathrm{R}$ of 0.76 [39] and 0.60 [36], respectively. As both studies of mild asthma patients used similar breath collection methodologies, these results might suggest that the e-nose profile better relates to small airway events, as represented by BAL sampling, but could be 

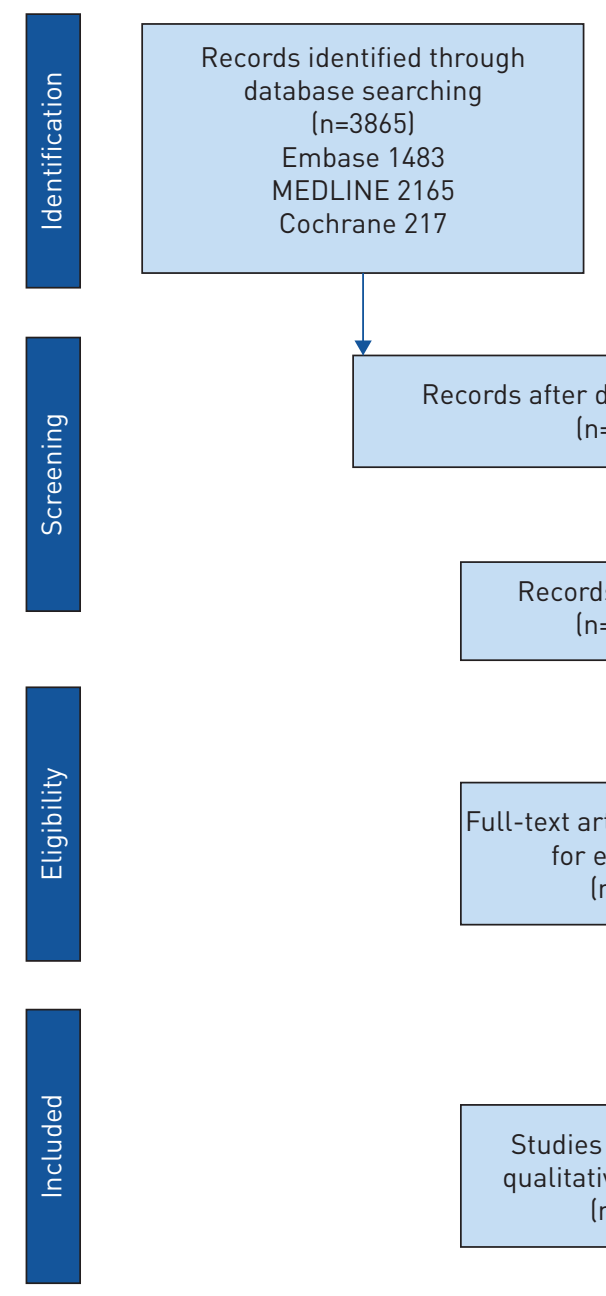

Records after duplicates removed ( $n=2957)$
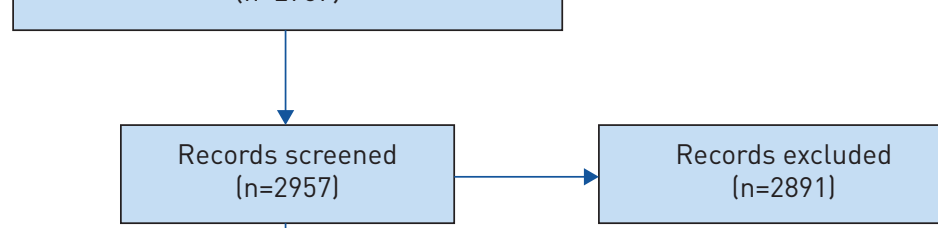

FIGURE 1 Flow diagram of PRISMA (Preferred Reporting Items for Systematic Reviews and Meta-Analyses)-oriented systematic search. VOC: volatile organic compound.

confounded by the fact that patients withheld steroid therapy before sputum, but not bronchoscopy sampling.

Three further studies assessed the ability of exhaled VOCs to discriminate inflammatory phenotype, defined by sputum granulocyte percentages. The e-nose discriminated sputum eosinophilia ( $3 \%$ cut-off) from paucigranular disease, with an area under the receiver operating characteristic curve (AUC) of 0.79 [46]. GCMS analysis discriminated eosinophilic (2\% cut-off) from non-eosinophilic sputum with an AUC of 0.98 [45]. A more extensive GCMS study was able to discriminate eosinophilic ( $3 \%$ cut-off) from paucigranulocytic and neutrophilic ( $76 \%$ cut-off) with an AUC of 0.99 and 0.92 , respectively [38]. Hoverer, it is not possible to reliably compare e-nose with GCMS approaches from these studies, due to separate breath-collection strategies and statistical analytical approaches. Critically, the Belgian study was able to test its model in a replication cohort demonstrating AUCs of 0.68 and 0.71 for eosinophilic versus paucigranulocytic and neutrophilic, respectively [38].

The ability of exhaled VOCs to discriminate patients by their inflammatory profile is further supported by unbiased cluster analysis of e-nose profiles of the U-BIOPRED cohort [47]. These clusters differed by systemic inflammatory profiles and anti-inflammatory medication profile use. They did not differ by sputum eosinophils or neutrophil percentages, but a change in sputum eosinophil percentages was seen in those patients that migrated clusters at 18 months. Using a different set of e-nose derived principal components, this study was also able to discriminate sputum transcriptomic associated clusters [54] in a small pilot analysis $(\mathrm{n}=28)$ [47].

\section{Treatment stratification}

In patients who had been withdrawn from their regular inhaled corticosteroid therapy, the e-nose breathprint was found to be accurate in predicting who would respond to an oral corticosteroid course 
TABLE 1 Summary of titles included, organised by clinical dilemma

\begin{tabular}{|c|c|c|c|}
\hline $\begin{array}{l}\text { First author (year) } \\
\text { [reference] }\end{array}$ & Design & Methodology & Brief summary of main findings \\
\hline
\end{tabular}

\section{Diagnosis}

DRAgonierI (2007) [40]

Cross-sectional

e-nose (Cyranose 320) GCMS

FENS (2009) [41]

Cross-sectional

e-nose (Cyranose 320)

MontuschI (2010) [42]

Cross-sectional

e-nose (Tor Vergata) GCMS

FEns (2011) [43]

TImms (2012) [34]

Cross-sectional

e-nose (Cyranose 320)

DE VRIES (2015) [21]

Cross-sectional

e-nose (Spironose)

DrAgonieRI (2019) [44]

Cross-sectional ${ }^{\#}$

e-nose (Cyranose 320)

\section{Phenotyping}

IBRAHIM (2011) [45]

Cross-sectional

GCMS

MeYer (2014) [37]

Plaza (2015) [46]

e-nose (Cyranose 320)

Fens (2015) [39]

BRINKMAN (2019) [47]
Cross-sectional

Cross-sectional

Cross-sectional

Longitudinal and cross-sectional ${ }^{\#}$
GC ToF MS e-nose breathprints accurately discriminate asthma from age-matched controls

Less successful at discriminating mild and severe asthma

Explorative GCMS analysis identified compounds in asthma

e-nose breathprints accurately discriminate asthma from COPD (smoking and nonsmoking as well as ICS-treated and non-ICS-treated)

e-nose breathprints accurately discriminate asthma from nonsmoking controls

e-nose breathprints accurately discriminate asthma from healthy controls

e-nose superior to $F_{\mathrm{eNO}}$ and spirometry for diagnostic accuracy

Late expiratory phase breath sampling (described as "alveolar air") gives better separation than mixed expiratory for the e-nose

No correlation between e-nose and $F_{\mathrm{eNO}}$ or spirometry

e-nose breathprints accurately discriminate asthma with fixed airways obstruction and reversible airway obstruction from COPD

Less successful at discriminating fixed airways obstruction from reversible airways obstruction

e-nose breathprint could discriminate asthma with GORD from healthy controls and asthma without GORD from healthy controls

Could also discriminate asthma from COPD

e-nose breathprints accurately discriminate asthma from COPD and healthy controls

Less successful at discriminating asthma from lung cancer

No significant difference in breathprints from asthma patients at different sites

e-nose breathprints accurately discriminate asthma with allergic rhinitis from allergic rhinitis (no asthma) and healthy controls

VOC model accurately discriminates asthma from healthy controls

VOC model accurately discriminates lusing sputum granulocyte percentages) eosinophilic from non-eosinophilic asthma (superior to $F_{\mathrm{eNO}}$ ) and neutrophilic from non-neutrophilic asthma phenotypes

VOC model accurately discriminates controlled from uncontrolled asthma (using ACQ score)

VOC model accurately discriminates asthma from healthy controls

Identified 7 clusters lusing 16 discriminatory VOCs and clinical parameters) with different clinical phenotypes

e-nose breathprints accurately discriminates lusing sputum granulocyte percentages) eosinophilic from neutrophilic asthma, eosinophilic from paucigranular asthma and neutrophilic from paucigranular asthma

e-nose breathprints associated with bronchoscopy lavage fluid eosinophil percentages (no relationship found between FeNO and BAL eosinophils)

Identified 3 clusters (using e-nose breathprints) with significant differences in chronic OCS usage and blood eosinophil and blood neutrophil percentages

(composite platform)
The majority of patients had migrated clusters at follow-up; patients that migrated clusters had changes in their sputum eosinophils 
TABLE 1 Continued

\section{First author (year)} [reference]
Cross-sectional ${ }^{\#}$

Schleich (2019) [38]
Design

\section{Exacerbation}

assessment/

prediction

OLOPADE (1997) [33]

BRINKMAN (2017) [50]

\section{Main findings not applicable to clinical categories}

LAZAR (2010) [51]
Methodology

Brief summary of main findings
Longitudinal and cross-sectional

Cross-sectional

Cross-sectional

Longitudinal and cross-sectional $^{\#}$

GC ToF MS

e-nose (Cyranose 320)

Breath ethanol device (Alcometer SD-400TM)

GC ToF MS

Longitudinal and cross-sectional

Longitudinal

Longitudinal and cross-sectional

e-nose (Cyranose 320)

e-nose lcomposite platform) and GCMS
VOC model accurately discriminates lusing sputum granulocyte percentages) eosinophilic from paucigranular asthma, eosinophilic from neutrophilic asthma and neutrophilic from paucigranular asthma

VOC model accurately discriminates eosinophilic from non-eosinophilic asthma (with similar accuracy to FeNO and blood eosinophils) and neutrophilic from non-neutrophilic asthma

VOC model unable to discriminate smokers, ex-smokers and nonsmokers

VOC model unable to discriminate ICS-treated and ICS-naïve patients

e-nose breathprints accurately discriminate asthma from healthy controls (maintained after asthma patients treated with oral prednisolone)

Accurately discriminates lat the point of full treatment withdrawal) patients who had lost control from ICS withdrawal from patients who had not

Accurately discriminates lat the point of full treatment withdrawal) patients who were OCS-responsive from OCS-unresponsive patients

e-nose breathprint correlates with sputum eosinophils

Exhaled ethane levels increased in asthma patients not receiving steroid therapy compared to steroid treated asthma patients and controls

Ethane concentrations increased in patients with more severe bronchoconstriction and gas trapping

Exhaled ethanol levels are transiently elevated following the use of metred dose inhalers using hydrofluoroalkane propellants

Exhaled VOCs associated with urinary detection of salbutamol and oral corticosteroids

Exhaled pentane concentrations increased in acute exacerbation compared to healthy controls (higher in those requiring admission from the emergency department)

Pentane concentration significantly decreased following treatment to concentrations similar to concentrations found in healthy controls and stable (outpatient) asthma

VOC model discriminates baseline from loss of control and loss of control from recovery

e-nose breathprint accurately discriminates baseline from loss of control and loss of control from recovery

GCMS identified compounds correlated with sputum eosinophil but not sputum neutrophils; e-nose breathprints did not correlate with either

e-nose breathprint discriminates post-methacholine from baseline and post-salbutamol (also post-methacholine) from baseline, but unable to discriminate post-methacholine and post-salbutamol lalso post methacholine)

e-nose breathprint discriminates post-saline from baseline and post-saline from post-salbutamol (also post-saline) 
TABLE 1 Continued

$\begin{array}{lll}\text { First author lyear) Design } \quad \text { Methodology } & \text { Brief summary of main findings }\end{array}$

[reference]

VAn der Schee (2013)

[52]

LÄRSTAD (2007) [53]
Cross-sectional GCMS

Cross-sectional
GC FID
Peak intensities of GCMS identified compounds did not show changes related to storage time (of up to 14 days)

VOC model and e-nose breathprints discriminate asthma from healthy controls with similar accuracies following different storage times

Isoprene and pentane concentrations increase with breath-holding

Ethane concentrations decreased at higher flow rates; pentane increased

Studies are categorised according to their main aim. The main aims of the studies are briefly summarised and are by no means exhaustive. GCMS: gas chromatography-mass spectrometry; COPD: chronic obstructive pulmonary disease; ICS: inhaled corticosteroids; FeNO: exhaled nitric oxide fraction; GORD: gastro-oesophageal reflux disease; VOC: volatile organic compound; ACQ: Asthma Control Questionnaire; GC ToF MS: gas chromatography-time-of-flight mass spectrometry; BAL: bronchoalveolar lavage; OCS: oral corticosteroids; GC-FID: gas chromatography with flame-ionisation detection. " : external validation of results.

(AUC=0.88), defined as a $\geqslant 12 \%$ increase in forced expiratory volume in $1 \mathrm{~s}(\mathrm{FEV} 1)$ and/or two or more doubling doses of inhaled AMP to cause a 20\% decline in FEV1 [36]. While the steroid-responsive group $(\mathrm{n}=11)$ had higher FeNO and sputum eosinophil measurements at baseline, as compared to the steroid-unresponsive group $(n=9)$, the respective predictive AUCs of 0.55 and 0.61 , respectively, for these measures were not as good as that for e-nose [36]. Other than in the aforementioned steroid withdrawal studies, we found no studies designed to look at treatment responders versus nonresponders.

\section{Treatment monitoring}

E-nose-derived clusters differ by oral corticosteroid use [47], and exhaled pentane levels are reduced in patients receiving inhaled corticosteroid treatments [48], consistent with the premise that VOCs are sensitive to ongoing inflammation. However, while oral corticosteroid use weakens the diagnostic (asthma versus healthy controls) accuracy of FeNO, this is not the case for the e-nose breathprint [36]. Following oral corticosteroid treatment of asthma patients, the e-nose continued to be able to diagnose asthma, suggesting discrimination independent of ongoing inflammation, possibly related to the medication itself. Similarly, the discrepancy between e-nose VOC profiles and BAL and sputum eosinophil percentages [36, 39], may be explained by treatment effect: if the VOC profile were sensitive to eosinophilic inflammation alone, a better relationship would be expected without the suppressive and noise effect of steroid therapy. Consistent with this, the U-BIOPRED group have reported that exhaled VOCs correlate with urinary levels of salbutamol and oral corticosteroid (by liquid chromatography-high resolution mass spectrometry) [49], confirming that some exhaled VOCs are related to asthma medications.

\section{Exacerbation prediction}

Markers of lipid peroxidation are elevated in acute exacerbations before returning to normal levels following treatment [33]. In addition, they are increased in patients with more severe disease (as defined by more reduced lung function) [48]. Principal components derived from e-nose breathprints can discriminate episodes of stability (baseline or at recovery with oral corticosteroids) from episodes of loss of control, due to treatment withdrawal. While exhaled VOCs have been measured prospectively in children to predict exacerbation events [55], no such studies have been performed in adults. In the two steroid withdrawal studies captured in this systematic review, one did not measure exhaled VOCs at baseline [36], and in the other, all but one patient suffered a loss of control on treatment withdrawal [50]. Therefore, these studies were unable to compare exacerbators against non-exacerbators.

\section{Breath sampling}

Concerning sampling, storage and analysis, few studies use the same strategies. Although a degree of consistency is seen from the studies originating from the same group [21, 40,41, 43, 50-52], wide methodological variation is seen at every step in exhaled breath methodology. This is illustrated in table 2 and expanded upon in the supplementary material.

Most studies instruct patients to perform a single vital capacity manoeuvre: from maximal inspiration to maximal expiration. The majority of these studies collect VOCs from this total expiratory phase, as it is 
TABLE 2 Methodological variation across studies split by breath fraction from which volatile organic compounds were captured

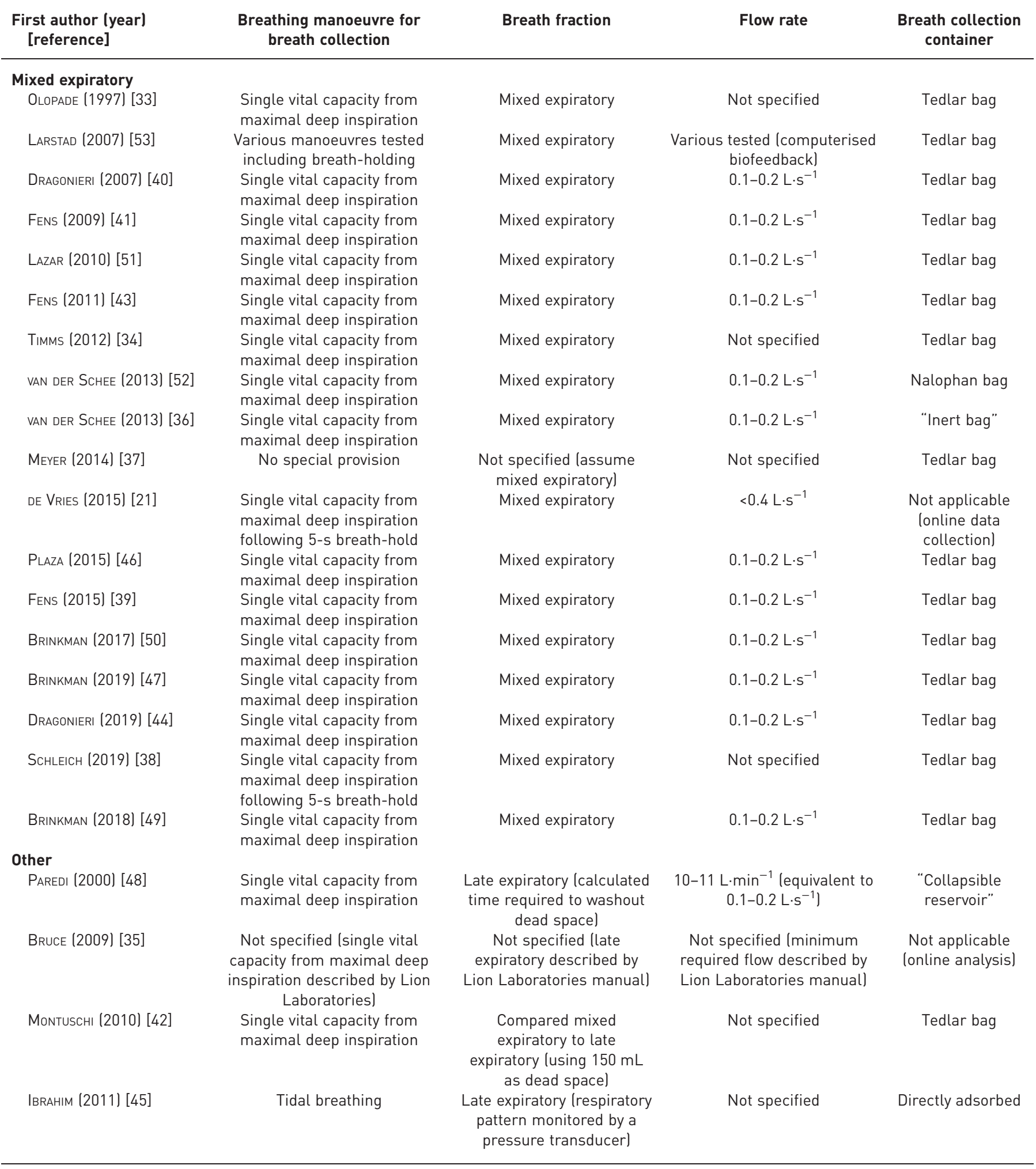

logistically most simple to achieve, but is contaminated by air within the oropharynx. Several studies have excluded dead space using strategies ranging from the crude (valves and estimated volumes [56, 57]), to highly engineered (pressure sensors [58]). 
Just 13 studies measured exhaled flow rate. 12 of these regulated exhaled breath flow between 0.1 and $0.2 \mathrm{~L} \cdot \mathrm{s}^{-1}$, which minimises nasal contamination of the sample by ensuring closure of the soft palate $[59,60]$, rather than due to a determination of optimal VOC capture. Most studies collect breath samples in an inert polymer, usually Tedlar, bag followed by storage onto thermal desorption tubes containing some adsorbent material, usually Tenax. Once the exhaled breath is collected, it can be either analysed "online" or "offline". Online refers to real-time analysis, but other than the Alcometer, a blood alcohol "breathalyser" [35], no studies have used such a platform.

\section{VOC analysis}

Since its first description in 2007 [40], the majority of modern studies utilise e-nose technology. Preprocessing procedures on raw data is described in few studies, and even then only briefly [38, 47]. Variation in statistical methodology, ranging from univariate to machine-learning techniques (table 3) broadly illustrates evolution over time: statistical analysis now involves some form of dimension-reduction strategy coupled to some form of machine-learning classifier. Several studies used "leave one out cross-validation techniques" but, until recently, external validation was notable by its rarity.

\section{Risk of bias}

In this systematic review, the QUADAS-2 scoring system highlights concerns in the published literature across several domains [32] (figure 2). Many studies applied smoking history, use of oral corticosteroids (due to the severity of illness) and common comorbidities as exclusion criteria to patient recruitment. By removing diagnostically challenging patients, studies are likely to provide over-optimistic discriminatory estimates. In addition, poor reference standards might undermine published results. Examples include diagnostic studies using asthma diagnosis based on physician assessment only [61] and inflammatory phenotyping studies based on sputum granulocyte percentages [62] or gastro-oesophageal reflux disease diagnosis without objective evidence. However, the most significant source of bias related to the analysis of exhaled VOC data (both GCMS or e-nose). In the absence of a clear consensus on the best statistical approach to multivariate data and an over-reliance on internal validation, the statistical findings described by authors are likely to be over-optimistic [63].

The flow and timing between index test and reference standards did not clearly introduce any bias, and the applicability of the identified studies to our review question was generally very good. The applicability of some study designs, such as cluster analysis, to our review question, which relates to clinical applications, was not clear. Similarly, studies relying on GCMS-based analysis techniques for acute assessment were found to be of limited translation potential.

\section{Discussion}

Technological evolution

Reviewing the past few decades of asthma-VOC literature demonstrates the rapid technological evolution of this field. Early breath research was limited to a "bottom-up" approach: VOCs were targeted a priori and analysed using expensive and laborious chemical techniques. Consequently, these studies were limited in numbers and focused on markers of oxidative stress [33] (inflammation not specific to asthma [48, 64]). The modern parallel developments of improved separation techniques, improved lower limits of detection, e-nose technology and high-throughput omics analysis platforms allow the full spectrum of exhaled VOCs to be analysed "top-down" as highly dimensional composite profiles: "breathomics".

Modern breathomics can refer to several VOC analysis platforms [18, 65], but mass spectrometry continues to be the gold standard [66], due to its ability to identify composition and concentrations of individual VOCs. However, it is too expensive in terms of expertise and equipment. Although the e-nose sensor array is cheaper, quicker and easier to use [40,57], it sacrifices that ability to reliably trace back to analytes of interest [67]. For clinicians, the focus on "treatable traits" [7, 68] requires a probabilistic approach: stratification of a patient based upon a clinically relevant quality (diagnosis, prognosis, treatment response) [69]. Understanding the mechanisms behind that biomarker/biomarker profile is not necessary [70], so sensor-based systems are ideally suited. In contrast, if the biomarker is being interrogated for mechanistic purposes [71] (e.g. drug target discovery), then the costs of chemical analytic techniques are likely justifiable. That is not to say that the two technologies are mutually exclusive: both can be successfully integrated into the same study [31], and improved understanding of the mechanisms and factors contributing to VOC profiles could inform future sensor-based technologies.

\section{Breath collection heterogeneity}

Of course, the strength of both platforms is intimately linked with the quality of its reference library. Efforts to build these libraries are already underway [72, 73] (www.breathomix.com/breathbase-data) and will only improve as more studies engage with such processes. Critically, such libraries can only be 
TABLE 3 Statistical approaches to exhaled volatile organic compound (VOC) data, organised by type of data

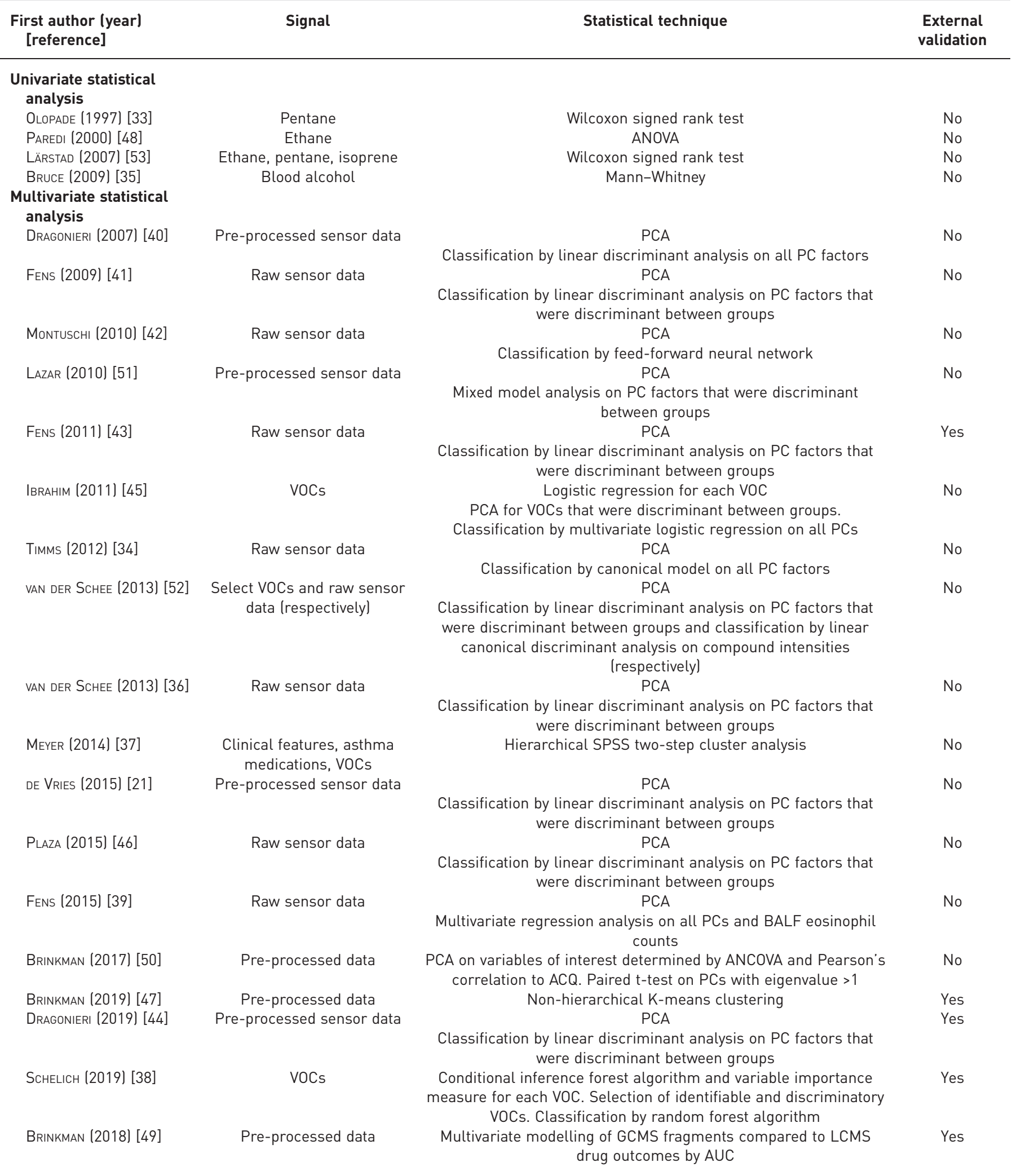

PCA: principal component analysis; PC: principal component; BALF: bronchoalveolar lavage fluid; ACQ: Asthma Control Questionnaire; GCMS: gas chromatography-mass spectrometry; LCMS: liquid chromatography-mass spectrometry; AUC: area under the receiver operating characteristic curve. 
a)

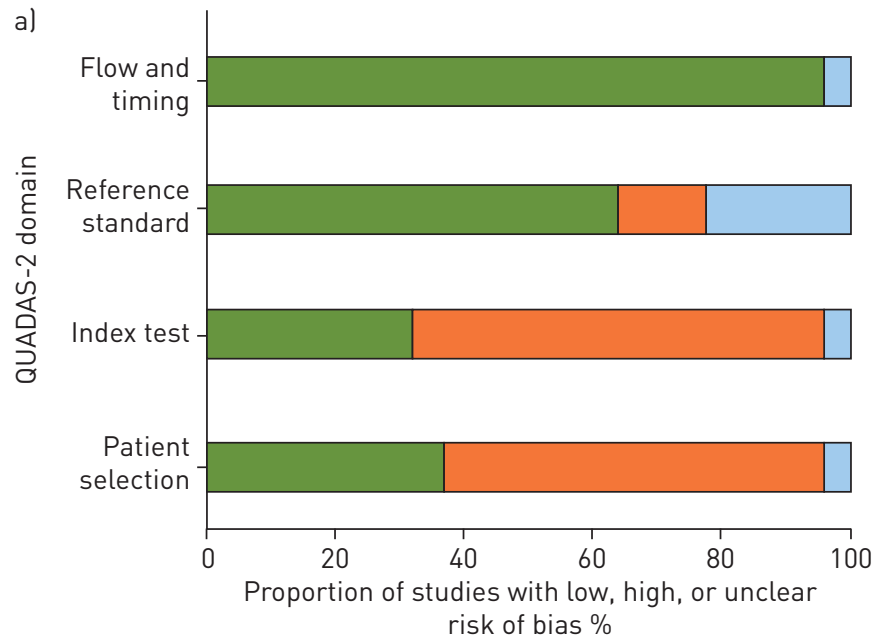

b)

$\square$ Low $\square$ High $\square$ Unclear

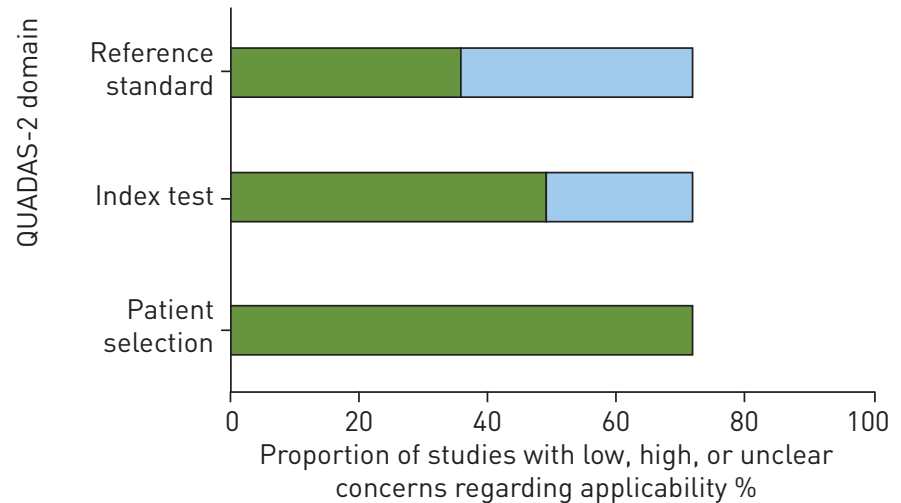

FIGURE 2 Quality assessment of diagnostic accuracy studies (QUADAS)-2 graphical display. a) Risk of bias; b) applicability concerns.

referenced against when using the same methodological parameters, so sharing meta-data relating to sample handling, processing and analysis is of paramount importance. The methodological heterogeneity of the breath analysis literature is well documented [30,74]. While exhaled breath sampling is simple for patients, by definition, the analyte of interest is volatile, and each stage of exhaled breath analysis introduces additional sources of variation [74].

Like exhaled nitric oxide concentrations [60,75], individual VOC concentrations [53] and e-nose sensor deflections [21] can be influenced by flow rate and breath-holding [21]. Similarly, most studies collect VOCs from the total expiratory phase, as it is logistically most simple to achieve. E-nose breathprints using this breath sample show more inferior diagnostic potential (asthma versus healthy controls) than when excluding air within the oropharynx [42], possibly reflecting the dilution of discriminatory VOCs by this contaminant air. There has been a range of strategies used to exclude air from this dead space, including valves and estimated volumes, which are inconsistent and unreliable [56,57], to a highly engineered system of pressure sensors $[45,58,76]$, which is expensive and bulky. The optimal solution needs to balance practicality and precision, so as not to negate the clinical utility of breath sampling. The breath sampler developed by a broad consortium of breath researchers and engineers (www.breathe-free.org), represents one such solution. A similar pragmatism is likely necessary for the exclusion of exogenous VOCs. Subtracting ambient VOC concentrations from exhaled VOC concentrations (alveolar gradient) [77] risks the loss of salient signals and ignores VOC interactions within the airways $[78,79]$. Although unable to eliminate all exogenous VOCs, filters can at least reduce background contamination [40].

Once collected, unless performing online analysis, the storage medium bears consideration. Most early studies stored breath samples in an inert polymer (usually Tedlar) bag, but the concentrations of compounds stored in Tedlar bags show compound-specific decay rates and the bags themselves can introduce contaminants [80, 81]. VAN DER SCHEE et al. [52] found no variation when breath samples were stored for up to 2 weeks, and many studies try to minimise storage time [40, 42]. Alternative or subsequent storage solutions: thermal desorption tubes containing some sort of adsorbent material (porous organic polymers, activated charcoal, carbon molecular sieves or graphitised carbon blacks) do not guarantee against this decay [81]. No adsorptive material can completely capture all the VOCs in the breath without some degree of loss [82].

Moreover, different materials are vulnerable to breakthrough (non-quantitative adsorption of analytes) and memory effect (incomplete desorption resulting in interference with subsequent measures) [83]. Therefore, ideally, the choice of adsorbent materials and the duration of storage [52] should be determined by compounds of interest [82, 84]. Most studies now adsorb onto Tenax TA (2,6-diphenyl-p-phenylene oxide) [85], due to its hydrophobicity, thermal stability and its ability to absorb a wide range of VOCs [86].

\section{Data handling heterogeneity}

Even then, a robust and reliable sample collection is relatively straightforward when compared to data management, analysis, and interpretation [87]. There are many thousands of VOCs in exhaled breath [13], and a proportion of these will relate to non-disease-related factors: age [88], sex [89], diet [90], exercise 
[91] and smoking [92], as well as therapies [51, 93], the resident microbiome [18] and environmental exposures [70, 81, 94-96]. Furthermore, it is also appreciated that not all VOCs originate from the airways. Non-asthma-VOC research proposes a model of blood/gas coefficients [97] and quantification of regional lung ventilation and perfusion [98] to describe the delivery and migration of systemically generated VOCs to exhaled breath.

Almost all the identified discriminatory VOCs identified in asthma studies are straight-chain, branched or aromatic hydrocarbons [81], and while there are proposed endogenous origins to such compounds [79], they frequently occur exogenously [99] and may simply reflect differential uptake of environmental VOCs [100]. Consequently, those VOCs that occur commonly and in high concentrations or those that are common to many inflammatory states $[64,101]$, are over-represented in the literature. For airways diseases, it is likely that actually, only a fraction of the VOCs identified in exhaled breath actually relate to airway events.

The majority of studies in this review explore their data using principal component analysis (PCA). Dimension reduction in this manner usefully allows focus on the variation of interest [28]. Classification by only using discriminatory principal components results in better diagnostic classification (asthma versus healthy controls) than when using all principal components unselectively [42]. Different principal components are used to discriminate diseases and inflammatory phenotype [39, 45], just as e-nose discrimination of transcriptomic-associated clusters [54] in the U-BIOPRED study relies on different principal components to those used to cluster patients according to inflammation [47]. As such, the VOCs used to discriminate across sputum granulocyte phenotypes were unable to discriminate between inhaled corticosteroid (ICS)-treated or ICS-naïve patients [38]. The disadvantage of PCA is its sensitivity to outliers, the handling of which is critical but rarely commented upon [102]. Moreover, data restructuring in this manner abstracts the relationship between trait/mechanism and biomarker.

For supervised statistical analyses, we commonly see linear discriminant analysis and partial least squares discriminant analysis, both of which investigate linear relationships. Complex biological systems are characterised by non-linear relationships, and although non-linear statistical techniques deliver the highest accuracy, their application may be limited by difficulty in their interpretation [103]. In the absence of a clear consensus on the optimal statistical approach, studies are likely to publish highly internally valid results which, in the absence of external validation, probably overestimate real-world findings [63]. This influence of data handling on biomarker identification means that transparency is more important than ever. The TRIPOD (Transparent Reporting of a Multivariable Prediction Model for Individual Prognosis or Diagnosis) recommendations on reporting multivariable prediction models [104] and STARD (Standards for Reporting of Diagnostic Accuracy Studies) guidelines on reporting of diagnostics accuracy studies [105] provide useful frameworks for future publications.

\section{Limitations of systematic review}

We specifically sought to describe biomarkers for precision medicine in asthma [7]; however, few studies specifically measure underlying biology or treatable traits, focusing instead on historical diagnostic labels [106]. In addition, we found that studies focused on milder cohorts, poorly representative of the heterogeneity of asthma, which is greatest in those with severe disease [1,2,107, 108]. Although we deliberately excluded paediatric studies, they have been summarised elsewhere [31]. Our broad and inclusive search strategy successfully captures the full spectrum of study designs, populations and outcome measures seen in exhaled VOC research. While this undermines some of the QUADAS risks-of-bias scores, we believe that our search allows for a comprehensive review of this emerging field.

\section{Future considerations}

It is evident that the field of breathomics is addressing the limitations mentioned herein. The breath research community has developed a framework for methodological standardisation $[30,109]$ and the latest breathomics findings are coming from large, multicentre studies with defined validation cohorts [22, 38, 47]. Large-scale studies, which recruit over a protracted period and over multiple sites [81] introduce new challenges. Such studies demand a multibatch experimental design (also unavoidable due to limitations of VOC storage solutions). Regular instrument proficiency testing and the use of quality control samples [110] is critical for demonstrating consistent quality assurance throughout such a study. Metabolomics studies demand standard operating protocols for both the analytical and computational workflows [81], including strategies to monitor within- and between-batch measurement variations [111]. This extends to data preprocessing of MS data (denoising and baseline correction, alignment, peak picking and merging of the peaks) as well as normalisation, scaling and transformation of the data, essential to constructing a reliable data matrix [103]. Consistent with previous reviews, we found that details of this were rarely reported [63], although recent publications are reversing this trend $[38,47]$. The ultimate intention for breathomics 
research would be interlaboratory and equipment comparison [112]. Only then will true external validation (where findings are replicated in a new study) be possible [113].

Robust biomarkers for T2-low asthma remain elusive [71, 114-116] despite these patient groups being most treatment-resistant and having the worst clinical outcomes [2, 117, 118]. The airway microbiome and host-microbial interactions have been proposed as a way to understand these poorly characterised asthma endotypes [119, 120]. Where bacteria are abundant producers of VOCs [121-123], they are likely to contribute to the spectra of exhaled VOCs. E-nose breathprints have been able to specifically discriminate patients colonised with or without potentially pathogenic bacteria in COPD [124], and the same is likely to be the case for asthma. For example, nonanal, identified to discriminate neutrophilic from eosinophilic asthma [38], is produced by bacterial species and has antimicrobial properties of its own [125], which may, in turn, contribute to the reduction in microbial diversity seen in neutrophilic asthma [126].

As airways diseases move closer to precision medicine, study designs will also have to consider the best way to assess VOCs. Biomarker studies are only as good as the gold standard to which they are compared. Existing gold standard biomarkers, such as FeNO and blood eosinophils, may not accurately predict T2-high biology [62]. Failings of a new biomarker (such as VOCs) may, in fact, reflect imperfect gold standard tests [127]: e-nose, for example, can discriminate (sputum) transcriptomic associated clusters (TAC clusters) [47] more effectively than existing biomarkers. Therefore, studies that aim to assess the biomarker potential of breathomics should consider moving away from solely comparing to these existing biomarkers and towards the direct assessment of biology, possibly alongside other omics platforms [128]. GCMS studies might also consider parallel in vitro studies as a means to validate in vivo findings [129, 130]. Alternatively, the focus should be on crucial disease traits such as treatment response [69], exacerbation prediction [28] and treatment adherence [131, 132]. Early evidence shows e-nose to demonstrate superior accuracy at predicting clinical response to steroids (compared to FeNO or sputum eosinophils) [36, 42], as well as accurate GCMS detection of VOCs, linked to salbutamol and OCS use [49].

\section{Conclusions}

Breathomics is still a relatively new field, and the guarded optimism with which findings are treated is justified (figure 2). As with other omics technologies, breathomics generally suffers from a lack of external validation, compounded by limited patient numbers and the risk of false discoveries due to model overfitting [12, 103, 113]. Nevertheless, the sensitivity of exhaled VOCs to underlying inflammation is corroborated across several studies using both sensor- and chemical-based platforms. Encouragingly, we see successful discrimination of asthma patients based upon their inflammatory phenotypes in a large replication cohort [38].

Strictly, true external validation has still not yet been met: the discriminatory VOCs in this study were not the same as those in a similar study [45] nor relevant in vitro work [130]. These might be explained away by differences in study design/methodology and the failings of headspace samples to accurately reflect complex interactions within the airways [133], but equally demonstrate the scale of what is still unknown in breathomics. Immediate priorities for breathomics include standardisation of reporting, a better understanding of the best statistical approaches and access to data-sharing platforms. Armed with these tools and study designs that maximise biomarker discovery, the next generation of breathomics evidence should show the full potential of exhaled VOCs.

Conflict of interest: A. Azim has nothing to disclose. C. Barber has nothing to disclose. P. Dennison has nothing to disclose. J. Riley is employed by and has shares in GSK. P. Howarth works for GSK in an educational role and has shares in GSK.

\section{References}

1 Moore WC, Meyers DA, Wenzel SE, et al. Identification of asthma phenotypes using cluster analysis in the Severe Asthma Research Program. Am J Respir Crit Care Med 2010; 181: 315-323.

2 Hinks TS, Brown T, Lau LC, et al. Multidimensional endotyping in patients with severe asthma reveals inflammatory heterogeneity in matrix metalloproteinases and chitinase 3-like protein 1. J Allergy Clin Immunol 2016; 138: 61-75.

3 Pavord ID, Korn S, Howarth P, et al. Mepolizumab for severe eosinophilic asthma (DREAM): a multicentre, double-blind, placebo-controlled trial. Lancet 2012; 380: 651-659.

$4 \quad$ Wenzel S, Ford L, Pearlman D, et al. Dupilumab in persistent asthma with elevated eosinophil levels. $N$ Engl J Med 2013; 368: 2455-2466.

5 Green RH, Brightling CE, McKenna S, et al. Asthma exacerbations and sputum eosinophil counts: a randomised controlled trial. Lancet 2002; 360: 1715-1721.

6 Chung KF. Defining phenotypes in asthma: a step towards personalized medicine. Drugs 2014; 74: 719-728.

7 Agusti A, Bel E, Thomas M, et al. Treatable traits: toward precision medicine of chronic airway diseases. Eur Respir J 2016; 47: 410-419.

8 Tiotiu A. Biomarkers in asthma: state of the art. Asthma Res Pract 2018; 4: 10.

9 Gisbert JP, Pajares JM. Review article: ${ }^{13} \mathrm{C}$-urea breath test in the diagnosis of Helicobacter pylori infection - a critical review. Aliment Pharmacol Ther 2004; 20: 1001-1017. 
Kharitonov SA, Yates D, Robbins RA, et al. Increased nitric oxide in exhaled air of asthmatic patients. Lancet 1994; 343: 133-135.

Nassar BS, Schmidt GA. Capnography during critical illness. Chest 2016; 149: 576-585.

Fowler SJ. Breath analysis for label-free characterisation of airways disease. Eur Respir J 2018; 51: 1702586.

Phillips M, Gleeson K, Hughes JM, et al. Volatile organic compounds in breath as markers of lung cancer: a cross-sectional study. Lancet 1999; 353: 1930-1933.

Phillips M. Breath tests in medicine. Sci Am 1992; 267: 74-79. partition chromatography. Proc Natl Acad Sci USA 1971; 68: 2374-2376.

Haick H, Broza YY, Mochalski P, et al. Assessment, origin, and implementation of breath volatile cancer markers. Chem Soc Rev 2014; 43: 1423-1449.

van de Kant KD, van der Sande LJ, Jöbsis Q, et al. Clinical use of exhaled volatile organic compounds in pulmonary diseases: a systematic review. Respir Res 2012; 13: 117.

van der Schee MP, Paff T, Brinkman P, et al. Breathomics in lung disease. Chest 2015; 147: 224-231.

Wilson AD. Advances in electronic-nose technologies for the detection of volatile biomarker metabolites in the human breath. Metabolites 2015; 5: 140-163.

Tisch U, Haick H. Chemical sensors for breath gas analysis: the latest developments at the Breath Analysis Summit 2013. J Breath Res 2014; 8: 027103.

de Vries R, Brinkman P, van der Schee MP, et al. Integration of electronic nose technology with spirometry: validation of a new approach for exhaled breath analysis. J Breath Res 2015; 9: 046001.

de Vries R, Dagelet YWF, Spoor P, et al. Clinical and inflammatory phenotyping by breathomics in chronic airway diseases irrespective of the diagnostic label. Eur Respir J 2018; 51: 1701817.

Mondello L, Tranchida PQ, Dugo P, et al. Comprehensive two-dimensional gas chromatography-mass spectrometry: a review. Mass Spectrom Rev 2008; 27: 101-124.

Smith D, Španěl P, Herbig J, et al. Mass spectrometry for real-time quantitative breath analysis. J Breath Res 2014; 8: 027101.

Mochalski P, Wiesenhofer $\mathrm{H}$, Allers $\mathrm{M}$, et al. Monitoring of selected skin- and breath-borne volatile organic compounds emitted from the human body using gas chromatography ion mobility spectrometry (GC-IMS). J Chromatogr B Analyt Technol Biomed Life Sci 2018; 1076: 29-34.

Bayrakli I. Breath analysis using external cavity diode lasers: a review. J Biomed Opt 2017; 22: 40901.

Rufo JC, Madureira J, Fernandes EO, et al. Volatile organic compounds in asthma diagnosis: a systematic review and meta-analysis. Allergy 2016; 71: 175-188.

Bos LD, Sterk PJ, Fowler SJ. Breathomics in the setting of asthma and chronic obstructive pulmonary disease. J Allergy Clin Immunol 2016; 138: 970-976.

Chung KF, Wenzel SE, Brozek JL, et al. International ERS/ATS guidelines on definition, evaluation and treatment of severe asthma. Eur Respir J 2014; 43: 343-373.

Horváth I, Barnes PJ, Loukides S, et al. A European Respiratory Society technical standard: exhaled biomarkers in lung disease. Eur Respir J 2017; 49: 1600965.

Neerincx AH, Vijverberg SJH, Bos LDJ, et al. Breathomics from exhaled volatile organic compounds in pediatric asthma. Pediatr Pulmonol 2017; 52: 1616-1627.

Whiting PF, Rutjes AW, Westwood ME, et al. QUADAS-2: a revised tool for the quality assessment of diagnostic accuracy studies. Ann Intern Med 2011; 155: 529-536.

Olopade CO, Zakkar M, Swedler WI, et al. Exhaled pentane levels in acute asthma. Chest 1997; 111: 862-865.

Timms C, Thomas PS, Yates DH. Detection of gastro-oesophageal reflux disease (GORD) in patients with obstructive lung disease using exhaled breath profiling. J Breath Res 2012; 6: 016003.

Bruce C, Chan HP, Mueller L, et al. Effect of hydrofluoroalkane-ethanol inhalers on estimated alcohol levels in asthmatic subjects. Respirology 2009; 14: 112-116.

van der Schee MP, Palmay R, Cowan JO, et al. Predicting steroid responsiveness in patients with asthma using exhaled breath profiling. Clin Exp Allergy 2013; 43: 1217-1225.

Meyer N, Dallinga JW, Nuss SJ, et al. Defining adult asthma endotypes by clinical features and patterns of volatile organic compounds in exhaled air. Respir Res 2014; 15: 136.

Schleich FN, Zanella D, Stefanuto PH, et al. Exhaled volatile organic compounds are able to discriminate between neutrophilic and eosinophilic asthma. Am J Respir Crit Care Med 2019; 200: 444-453.

Fens N, van der Sluijs KF, van de Pol MA, et al. Electronic nose identifies bronchoalveolar lavage fluid eosinophils in asthma. Am J Respir Crit Care Med 2015; 191: 1086-1088.

Dragonieri S, Schot R, Mertens BJ, et al. An electronic nose in the discrimination of patients with asthma and controls. J Allergy Clin Immunol 2007; 120: 856-862.

Fens N, Zwinderman AH, van der Schee MP, et al. Exhaled breath profiling enables discrimination of chronic obstructive pulmonary disease and asthma. Am J Respir Crit Care Med 2009; 180: 1076-1082.

Montuschi P, Santonico M, Mondino C, et al. Diagnostic performance of an electronic nose, fractional exhaled nitric oxide, and lung function testing in asthma. Chest 2010; 137: 790-796.

Fens N, Roldaan AC, van der Schee MP, et al. External validation of exhaled breath profiling using an electronic nose in the discrimination of asthma with fixed airways obstruction and chronic obstructive pulmonary disease. Clin Exp Allergy 2011; 41: 1371-1378.

Dragonieri S, Quaranta VN, Carratu P, et al. Exhaled breath profiling by electronic nose enabled discrimination of allergic rhinitis and extrinsic asthma. Biomarkers 2019; 24: 70-75.

Ibrahim B, Basanta M, Cadden P, et al. Non-invasive phenotyping using exhaled volatile organic compounds in asthma. Thorax 2011; 66: 804-809.

Plaza V, Crespo A, Giner J, et al. Inflammatory asthma phenotype discrimination using an electronic nose breath analyzer. J Investig Allergol Clin Immunol 2015; 25: 431-437.

Brinkman P, Wagener AH, Hekking PP, et al. Identification and prospective stability of electronic nose (eNose)-derived inflammatory phenotypes in patients with severe asthma. J Allergy Clin Immunol 2019; 143: $1811-1820$ 

Med 2000; 162: 1450-1454

49 Brinkman P, Ahmed W, Gómez C, et al. Exhaled volatile organic compounds as markers for medication use in asthma within the U-BIOPRED cohort. Am J Respir Crit Care Med 2018; 197: A5954.

50 Brinkman P, van de Pol MA, Gerritsen MG, et al. Exhaled breath profiles in the monitoring of loss of control and clinical recovery in asthma. Clin Exp Allergy 2017; 47: 1159-1169.

51 Lazar Z, Fens N, van der Maten J, et al. Electronic nose breathprints are independent of acute changes in airway caliber in asthma. Sensors 2010; 10: 9127-9138.

52 van der Schee MP, Fens N, Brinkman P, et al. Effect of transportation and storage using sorbent tubes of exhaled breath samples on diagnostic accuracy of electronic nose analysis. J Breath Res 2013; 7: 016002.

53 Lärstad MA, Torén K, Bake B, et al. Determination of ethane, pentane and isoprene in exhaled air - effects of breath-holding, flow rate and purified air. Acta Physiol 2007; 189: 87-98.

54 Kuo CS, Pavlidis S, Loza M, et al. T-helper cell type 2 (Th2) and non-Th2 molecular phenotypes of asthma using sputum transcriptomics in U-BIOPRED. Eur Respir J 2017; 49: 1603135.

55 Robroeks CM, van Berkel JJ, Jöbsis Q, et al. Exhaled volatile organic compounds predict exacerbations of childhood asthma in a 1-year prospective study. Eur Respir J 2013; 42: 98-106.

56 Miekisch W, Kischkel S, Sawacki A, et al. Impact of sampling procedures on the results of breath analysis. J Breath Res 2008; 2: 026007.

57 Cope KA, Watson MT, Foster WM, et al. Effects of ventilation on the collection of exhaled breath in humans. J Appl Physiol 2004; 96: 1371-1379.

58 Basanta M, Koimtzis T, Singh D, et al. An adaptive breath sampler for use with human subjects with an impaired respiratory function. Analyst 2007; 132: 153-163.

59 Kharitonov SA, Barnes PJ. Nasal contribution to exhaled nitric oxide during exhalation against resistance or during breath holding. Thorax 1997; 52: 540-544.

60 Silkoff PE, McClean PA, Slutsky AS, et al. Marked flow-dependence of exhaled nitric oxide using a new technique to exclude nasal nitric oxide. Am J Respir Crit Care Med 1997; 155: 260-267.

61 Aaron SD, Vandemheen KL, FitzGerald JM, et al. Reevaluation of diagnosis in adults with physician-diagnosed asthma. JAMA 2017; 317: 269-279.

62 Pavlidis S, Takahashi K, Ng Kee Kwong F, et al. “T2-high” in severe asthma related to blood eosinophil, exhaled nitric oxide and serum periostin. Eur Respir J 2019; 53: 1800938.

63 Leopold JH, Bos LDJ, Sterk PJ, et al. Comparison of classification methods in breath analysis by electronic nose. J Breath Res 2015; 9: 046002.

64 Scholpp J, Schubert JK, Miekisch W, et al. Breath markers and soluble lipid peroxidation markers in critically ill patients. Clin Chem Lab Med 2002; 40: 587-594.

65 Rattray NJ, Hamrang Z, Trivedi DK, et al. Taking your breath away: metabolomics breathes life in to personalized medicine. Trends Biotechnol 2014; 32: 538-548.

66 Schwoebel H, Schubert R, Sklorz M, et al. Phase-resolved real-time breath analysis during exercise by means of smart processing of PTR-MS data. Anal Bioanal Chem 2011; 401: 2079-2091.

67 Fens N, van der Schee MP, Brinkman P, et al. Exhaled breath analysis by electronic nose in airways disease. Established issues and key questions. Clin Exp Allergy 2013; 43: 705-715.

68 McDonald VM, Fingleton J, Agusti A, et al. Treatable traits: a new paradigm for 21st century management of chronic airway diseases: Treatable Traits Down Under International Workshop Report. Eur Respir J 2019; 53: 1802058.

69 Arron JR, Izuhara K. Asthma biomarkers: what constitutes a "gold standard"? Thorax 2015; 70: 105-107.

70 Xia J, Broadhurst DI, Wilson M, et al. Translational biomarker discovery in clinical metabolomics: an introductory tutorial. Metabolomics 2013; 9: 280-299.

71 Chung KF. Personalised medicine in asthma: time for action. Eur Respir Rev 2017; $26: 170064$

72 de Lacy Costello B, Amann A, Al-Kateb H, et al. A review of the volatiles from the healthy human body. $J$ Breath Res 2014; 8: 014001.

73 Lemfack MC, Gohlke BO, Toguem SMT, et al. mVOC 2.0: a database of microbial volatiles. Nucleic Acids Res 2018; 46: D1261-D1265

74 Lawal O, Ahmed WM, Nijsen TME, et al. Exhaled breath analysis: a review of "breath-taking" methods for off-line analysis. Metabolomics 2017; 13: 110.

75 American Thoracic Society, European Respiratory Society. ATS/ERS recommendations for standardized procedures for the online and offline measurement of exhaled lower respiratory nitric oxide and nasal nitric oxide, 2005. Am J Respir Crit Care Med 2005; 171: 912-930.

76 Basanta M, Jarvis RM, Xu Y, et al. Non-invasive metabolomic analysis of breath using differential mobility spectrometry in patients with chronic obstructive pulmonary disease and healthy smokers. Analyst 2010; 135: 315-320.

77 Phillips M. Method for the collection and assay of volatile organic compounds in breath. Anal Biochem 1997; 247: 272-278

78 Garca-Morin M, López-Sangüos C, Vázquez P, et al. Lactate dehydrogenase: a marker of the severity of vaso-occlusive crisis in children with sickle cell disease presenting at the emergency department. Hemoglobin 2016; 40: 388-391.

79 Miekisch W, Schubert JK, Noeldge-Schomburg GF. Diagnostic potential of breath analysis - focus on volatile organic compounds. Clin Chim Acta 2004; 347: 25-39.

80 Beauchamp J, Herbig J, Gutmann R, et al. On the use of Tedlar ${ }^{\infty}$ bags for breath-gas sampling and analysis. J Breath Res 2008; 2: 046001.

81 Ahmed WM, Brinkman P, Weda H, et al. Methodological considerations for large-scale breath analysis studies: lessons from the U-BIOPRED severe asthma project. J Breath Res 2018; 13: 016001.

82 Stein VB, Narang RS, Wilson L, et al. A simple, reliable method for the determination of chlorinated volatile organics in human breath and air using glass sampling tubes. J Anal Toxicol 1996; 20: 145-150.

83 Miekisch W, Schubert JK. From highly sophisticated analytical techniques to life-saving diagnostics: technical developments in breath analysis. Trends Anal Chem 2006; 25: 665-673. 
Dettmer K, Engewald W. Adsorbent materials commonly used in air analysis for adsorptive enrichment and thermal desorption of volatile organic compounds. Anal Bioanal Chem 2002; 373: 490-500.

Tangerman A, Meuwese-Arends MT, van Tongeren JH. New methods for the release of volatile sulfur compounds from human serum: its determination by Tenax trapping and gas chromatography and its application in liver diseases. J Lab Clin Med 1985; 106: 175-182.

Beale DJ, Jones OA, Karpe AV, et al. A review of analytical techniques and their application in disease diagnosis in breathomics and salivaomics research. Int J Mol Sci 2016; 18: 24.

Mardis ER. The \$1,000 genome, the \$100,000 analysis? Genome Med 2010; 2: 84 .

Phillips M, Cataneo RN, Greenberg J, et al. Effect of age on the breath methylated alkane contour, a display of apparent new markers of oxidative stress. J Lab Clin Med 2000; 136: 243-249.

Crane MA, Levy-Carrick NC, Crowley L, et al. The response to September 11: a disaster case study. Ann Glob Health 2014; 80: 320-331.

Bikov A, Paschalaki K, Logan-Sinclair R, et al. Standardised exhaled breath collection for the measurement of exhaled volatile organic compounds by proton transfer reaction mass spectrometry. BMC Pulm Med 2013; 13: 43 . Bikov A, Lazar Z, Schandl K, et al. Exercise changes volatiles in exhaled breath assessed by an electronic nose. Acta Physiol Hung 2011; 98: 321-328.

Cheng ZJ, Warwick G, Yates DH, et al. An electronic nose in the discrimination of breath from smokers and non-smokers: a model for toxin exposure. J Breath Res 2009; 3: 036003.

Gaugg MT, Engler A, Nussbaumer-Ochsner Y, et al. Metabolic effects of inhaled salbutamol determined by exhaled breath analysis. J Breath Res 2017; 11: 046004.

Amann A, Mochalski P, Ruzsanyi V, et al. Assessment of the exhalation kinetics of volatile cancer biomarkers based on their physicochemical properties. J Breath Res 2014; 8: 016003.

Wild CP. Complementing the genome with an "exposome": the outstanding challenge of environmental exposure measurement in molecular epidemiology. Cancer Epidemiol Biomarkers Prev 2005; 14: 1847-1850.

Pleil JD, Sheldon LS. Adapting concepts from systems biology to develop systems exposure event networks for exposure science research. Biomarkers 2011; 16: 99-105.

Lourenço C, Turner C. Breath analysis in disease diagnosis: methodological considerations and applications. Metabolites 2014; 4: 465-498.

Levitt MD, Ellis C, Furne J. Influence of method of alveolar air collection on results of breath tests. Dig Dis Sci 1998; 43: 1938-1945.

Chen M-L, Chen S-H, Guo B-R, et al. Relationship between environmental exposure to toluene, xylene and ethylbenzene and the expired breath concentrations for gasoline service workers. J Environ Monit 2002; 4: 562-566. Cao W, Duan Y. Breath analysis: potential for clinical diagnosis and exposure assessment. Clin Chem 2006; 52: $800-811$.

Paredi P, Kharitonov SA, Leak D, et al. Exhaled ethane, a marker of lipid peroxidation, is elevated in chronic obstructive pulmonary disease. Am J Respir Crit Care Med 2000; 162: 369-373. validating volatile promises. Trends Mol Med 2015; 21: 633-644.

Smolinska A, Hauschild AC, Fijten RR, et al. Current breathomics - a review on data pre-processing techniques and machine learning in metabolomics breath analysis. J Breath Res 2014; 8: 027105.

Collins GS, Reitsma JB, Altman DG, et al. Transparent reporting of a multivariable prediction model for individual prognosis or diagnosis (TRIPOD): the TRIPOD statement. Eur Urol 2015; 67: 1142-1151.

Cohen JF, Korevaar DA, Altman DG, et al. STARD 2015 guidelines for reporting diagnostic accuracy studies: explanation and elaboration. BMJ Open 2016; 6: e012799.

Vanfleteren LE, Kocks JW, Stone IS, et al. Moving from the Oslerian paradigm to the post-genomic era: are asthma and COPD outdated terms? Thorax 2014; 69: 72-79.

O'Neill S, Sweeney J, Patterson CC, et al. The cost of treating severe refractory asthma in the UK: an economic analysis from the British Thoracic Society Difficult Asthma Registry. Thorax 2015; 70: 376-378.

Hyland ME, Whalley B, Jones RC, et al. A qualitative study of the impact of severe asthma and its treatment showing that treatment burden is neglected in existing asthma assessment scales. Qual Life Res 2015; 24: 631-639.

Herbig J, Beauchamp J. Towards standardization in the analysis of breath gas volatiles. J Breath Res 2014; 8: 037101.

Nakhleh MK, Amal H, Jeries R, et al. Diagnosis and classification of 17 diseases from 1404 subjects via pattern analysis of exhaled molecules. ACS Nano 2017; 11: 112-125.

Dunn WB, Wilson ID, Nicholls AW, et al. The importance of experimental design and QC samples in large-scale and MS-driven untargeted metabolomic studies of humans. Bioanalysis 2012; 4: 2249-2264.

van Oort PM, Nijsen T, Weda $\mathrm{H}$, et al. BreathDx - molecular analysis of exhaled breath as a diagnostic test for ventilator-associated pneumonia: protocol for a European multicentre observational study. BMC Pulm Med 2017; 17: 1. experiments. Metabolomics 2006; 2: 171-196. Thomson NC. Novel approaches to the management of noneosinophilic asthma. Ther Adv Respir Dis 2016; 10: 211-234.

Go LH, Krefft SD, Cohen RA, et al. Lung disease and coal mining: what pulmonologists need to know. Curr Opin Pulm Med 2016; 22: 170-178.

Schleich F, Demarche S, Louis R. Biomarkers in the management of difficult asthma. Curr Top Med Chem 2016; 16: 1561-1573.

Fahy JV. Type 2 inflammation in asthma - present in most, absent in many. Nat Rev Immunol 2015; 15: 57-65. Moore WC, Hastie AT, Li X, et al. Sputum neutrophil counts are associated with more severe asthma phenotypes using cluster analysis. J Allergy Clin Immunol 2014; 133: 1557-1563.

Huang YJ, Marsland BJ, Bunyavanich S, et al. The microbiome in allergic disease: current understanding and future opportunities - 2017 PRACTALL document of the American Academy of Allergy, Asthma \& Immunology and the European Academy of Allergy and Clinical Immunology. J Allergy Clin Immunol 2017; 139: 1099-1110. 
Green BJ, Wiriyachaiporn S, Grainge C, et al. Potentially pathogenic airway bacteria and neutrophilic inflammation in treatment resistant severe asthma. PLoS ONE 2014; 9: e100645.

121 Schulz S, Dickschat JS. Bacterial volatiles: the smell of small organisms. Nat Prod Rep 2007; 24: 814-842.

122 Thorn RM, Reynolds DM, Greenman J. Multivariate analysis of bacterial volatile compound profiles for discrimination between selected species and strains in vitro. J Microbiol Methods 2011; 84: $258-264$.

123 Bos LD, Sterk PJ, Schultz MJ. Volatile metabolites of pathogens: a systematic review. PLoS Pathog 2013; 9: e1003311.

124 Sibila O, Garcia-Bellmunt L, Giner J, et al. Identification of airway bacterial colonization by an electronic nose in chronic obstructive pulmonary disease. Respir Med 2014; 108: 1608-1614.

125 Rajer FU, Wu H, Xie Y, et al. Volatile organic compounds produced by a soil-isolate, Bacillus subtilis FA26 induce adverse ultra-structural changes to the cells of Clavibacter michiganensis ssp. sepedonicus, the causal agent of bacterial ring rot of potato. Microbiology 2017; 163: 523-530.

126 Taylor SL, Leong LEX, Choo JM, et al. Inflammatory phenotypes in patients with severe asthma are associated with distinct airway microbiology. J Allergy Clin Immunol 2018; 141: 94-103.

127 Waikar SS, Betensky RA, Emerson SC, et al. Imperfect gold standards for biomarker evaluation. Clin Trials 2013; 10: 696-700.

128 De Meulder B, Lefaudeux D, Bansal AT, et al. A computational framework for complex disease stratification from multiple large-scale datasets. BMC Syst Biol 2018; 12: 60.

129 Davies MP, Barash O, Jeries R, et al. Unique volatolomic signatures of TP53 and KRAS in lung cells. Br J Cancer 2014; 111: 1213-1221.

130 Schleich FN, Dallinga JW, Henket M, et al. Volatile organic compounds discriminate between eosinophilic and neutrophilic inflammation in vitro. J Breath Res 2016; 10: 016006.

131 Gamble J, Stevenson M, McClean E, et al. The prevalence of nonadherence in difficult asthma. Am J Respir Crit Care Med 2009; 180: 817-822.

132 Murphy AC, Proeschal A, Brightling CE, et al. The relationship between clinical outcomes and medication adherence in difficult-to-control asthma. Thorax 2012; 67: 751-753.

133 King J, Unterkofler K, Teschl G, et al. A modeling-based evaluation of isothermal rebreathing for breath gas analyses of highly soluble volatile organic compounds. J Breath Res 2012; 6: 016005. 